\title{
TITLE: Molecules star in quantum movie
}

STANDFIRST: The quantum interference of large molecules can be recorded in real time thanks to state-of-the-art nanofabrication and nano-imaging technologies.

AUTHORS: Bum Suk Zhao and Wieland Schöllkopf

Quantum physics predicts a variety of counter-intuitive and hard-to-believe phenomena. These include, for instance, the entanglement of distant particles, which Einstein famously referred to as "spooky action at a distance". Another basic quantum effect, no less bizarre, is wave-particle duality: under some experimental conditions particles such as electrons and atoms behave as waves, whereas light - perhaps the mostly widely studied wave phenomenon in physics - often behaves as a stream of particles called photons. For decades the interference pattern observed in a Young's double-slit experiment, which dates back to 1807 , was considered to be clear evidence that light was a wave. However, the double-slit experiment can actually reveal both wave and particle behaviour at the same time. In particular, an interference pattern can build up even if there is only one photon or one electron in the apparatus at any one time.

Now, in Nature Nanotechnology, Markus Arndt of the University of Vienna and coworkers ${ }^{1}$ release a movie showing the build up of the double-slit interference pattern for phthalocyanine molecules, which have masses of 514 atomic mass units (amu), and derivatives of phthalocyanine molecules with masses of $1298 \mathrm{amu}$. The latter molecules are the largest quantum particles for which the build up of an interference pattern from single-particle events has been recorded. The cutting-edge nanotechnologies used by Arndt and co-workers - who are based at the University of Vienna, Tel Aviv University, the University of Basel and Karlsruhe Institute of Technology - should pave the way to quantum interference experiments with ever more massive and complex molecules.

The work by Arndt and co-workers is the latest highlight in a long series of experiments that started more than 80 years ago, after de Broglie had predicted that a particle with momentum $p$ would have a wavelength $\lambda=h / p$, where $h$ is the Planck constant. In 1927, Davisson and Germer demonstrated the wave nature of electrons by recording the diffraction of electrons from the surface of a nickel crystal. This was the first experimental evidence that a particle could behave like a wave because, according to Huygens' principle, diffraction is a wave-interference phenomenon: it cannot be explained by a particle model. Four years later Estermann and Stern recorded the diffraction patterns produced by helium atoms and hydrogen molecules 
scattering off the surface of a sodium chloride crystal ${ }^{2}$.

The first double-slit experiment with electrons was performed 30 years later, by Jönssen, using a copper grating with a period of $1 \mu \mathrm{m}$ (fig. 1a) $)^{3,4}$, and the build-up of the interference pattern when there was only one electron in the apparatus at a time was first recorded by a group in Bologna ${ }^{5}$ (1976) and later by a group at Hitachi ${ }^{6}$ (1989). Both the Bologna and Hitachi groups also made movies ${ }^{7,8}$ showing how the wave-like diffraction pattern gradually builds up in the spatial distribution of a large number of stochastically arriving single particles.

The diffraction of atomic and molecular beams from crystal surfaces proved to be a useful and versatile tool for surface science. However, this approach does not work if the molecules are fragile (because they will break up if they hit the surface) or if they interact too strongly with the surface (because they will undergo inelastic scattering processes that wipe out the coherence needed to form the interference pattern).

The angle through which particles are diffracted by a grating scales as the ratio of the de Broglie wavelength to the period of the grating. The de Broglie wavelength in an atomic or molecular beam is typically on the order of $100 \mathrm{pm}$ or less, and for the heavy molecules used in the Vienna experiment it can be as short as $\sim 5 \mathrm{pm}$.

Therefore, the grating period needs to be of the order of $100 \mathrm{~nm}$ or less to make the diffraction angle large enough to allow the diffraction peaks to be resolved. In a pioneering experiment in 1988, Dave Pritchard and co-workers at MIT observed the diffraction of a beam of sodium atoms by a gold grating with a period of $200 \mathrm{~nm}$ (ref. 9). This was the first time that a beam of atoms had been diffracted by a fabricated structure. Subsequently, free-standing gratings with a period of $100 \mathrm{~nm}$ have routinely been made by Tim Savas and Hank Smith, also of $\mathrm{MIT}^{10}$, and have been used by groups around the world in matter-wave experiments ${ }^{11}$.

In 1999, the Vienna group used such a grating (Fig. 1b) to observe the diffraction of $\mathrm{C}_{60}$ molecules $^{12}$, which have a mass of $720 \mathrm{amu}$, and in 2011 they observed the diffraction of $\mathrm{C}_{60}\left[\mathrm{C}_{12} \mathrm{~F}_{25}\right]_{10}$ molecules, which contain 430 atoms and have a mass of $6910 \mathrm{amu}$, making them the largest molecules ever to demonstrate wave-like behaviour ${ }^{13}$. However, they did not use a conventional grating in that experiment, instead they used a laser to create a standing light wave, which acted as a grating, and they observed the near-field diffraction patterns that were produced when beams of various large molecules were scattered by this optical grating. Observing far-field diffraction patterns for such large molecules is not possible with gratings of the type 
shown in Fig. 1b because the interactions between the molecules and the grating material would diminish the effective width of the slits in the grating too much ${ }^{14}$. Now Arndt and co-workers have laid the groundwork for studying the diffraction of large molecules by conventional gratings by taking advantage of advances in nanofabrication and nano-imaging.

First, they have made a new generation of free-standing 100-nm-period gratings with an unprecedented thickness of $10 \mathrm{~nm}$ (Fig. 1c). The interactions between the molecules and the grating material are reduced to a tolerable level in such a thin grating. Second, they have implemented a laser-desorption molecular-beam source that allows them to produce a beam of large, complex organic molecules; such beams cannot be prepared by a conventional oven source as the molecules would fragment because of the heat. Third, they have been able to use a form of fluorescence microscopy that offers resolution beyond the diffraction limit ${ }^{15}$ to detect diffraction patterns with single-molecule sensitivity and with a spatial resolution of $10 \mathrm{~nm}$. This detection scheme is at least four orders of magnitude more sensitive than conventional electron-impact ionization methods.

The results obtained by Arndt and coworkers demonstrate how nanotechnologies will enable matter-wave diffraction experiments to be performed with molecules that are larger, more massive or more complex than at present. Moreover, these experiments should provide new insights into the differences between the quantum and classical worlds.

Bum Suk Zhao is in the School of Nano-Bioscience and Chemical Engineering, Ulsan National Institute of Science and Technology, Ulsan, Korea 689-798. Wieland Schöllkopf is at the Fritz-Haber-Institut der Max-Planck-Gesellschaft, 14195 Berlin, Germany. e-mail: zhao@unist.ac.kr; wschoell@fhi-berlin.mpg.de

\section{References}

1. Juffmann, T. et al. Nature Nanotech. http://dx.doi.org/10.1038/nnano.2012.34 (2012).

2. Toennies J.P., Schmidt-Böcking, H., Friedrich, B. \& Lower, J.C.A. Annalen der Physik 523, 1045-1070 (2011).

3. Jönsson, C. Z. Phys. 161, 454-474 (1961).

4. Jönsson, C; Am. J. Phys. 42, 4-11 (1974).

5. Merli, P.G., Missiroli, G.F. \& Pozzi, G. Am. J. Phys. 44, 306-307 (1976). 
6. Tonomura, A., Endo, J., Matsuda, T., Kawasaki, T. \& Ezawa, H. Am. J. Phys. 57, 117-120 (1989).

7. http://www.bo.imm.cnr.it/users/lulli/downintel/electroninterfea.html

8. http://www.hitachi.com/rd/research/em/doubleslit.html

9. Keith, D.W., Schattenburg, M.L., Smith, H.I. \& Pritchard, D.E. Phys. Rev. Lett. 61, 15801583 (1988).

10. Savas, T.A., Shah, S.N., Schattenburg, M.L., Carter, J.M. \& Smith, H.I. J. Vac. Sci.

Technol. B, 13, 2732-2735 (1995).

11. Cronin A.D., Schmiedmayer J. \& Pritchard, D.E. Rev. Mod. Phys. 81, 1051-1129 (2009).

12. Arndt, M. et al. Nature 401, 680-682 (1999).

13. Gerlich, S. et al. Nature Commun. 2, 263 (2011).

14. Grisenti, R.E., Schöllkopf, W., Toennies, J.P., Hegerfeldt, G.C. \& Köhler, T. Phys. Rev. Lett. 83, 1755-1758 (1999).

15. Huang, B., Babcock H. \& Zhuang, X. Cell 143, 1047 - 1058 (2010). 
Figure caption: The evolution of matter-wave diffraction experiments with free-standing gratings. a, In 1961 a copper grating with a period of $1 \mu \mathrm{m}$ and a thickness of $500 \mathrm{~nm}$ was used to perform the first Young's slit experiment with electrons ${ }^{3}$. b, In 1999 a silicon nitride grating with a period of $100 \mathrm{~nm}$ and a thickness of $160 \mathrm{~nm}$ was used to observe $\mathrm{C}_{60}$ diffraction patterns ${ }^{9}$. c, Now Arndt and co-workers ${ }^{1}$ have used a silicon nitride grating with a period of $100 \mathrm{~nm}$ and a thickness of just $10 \mathrm{~nm}$ to perform a Young's slit experiment with molecules that are bigger, heavier and more complex than $\mathrm{C}_{60}$, including the phthalocyanine derivative shown here. Moreover, they observed the interference pattern build up molecule by molecule. 


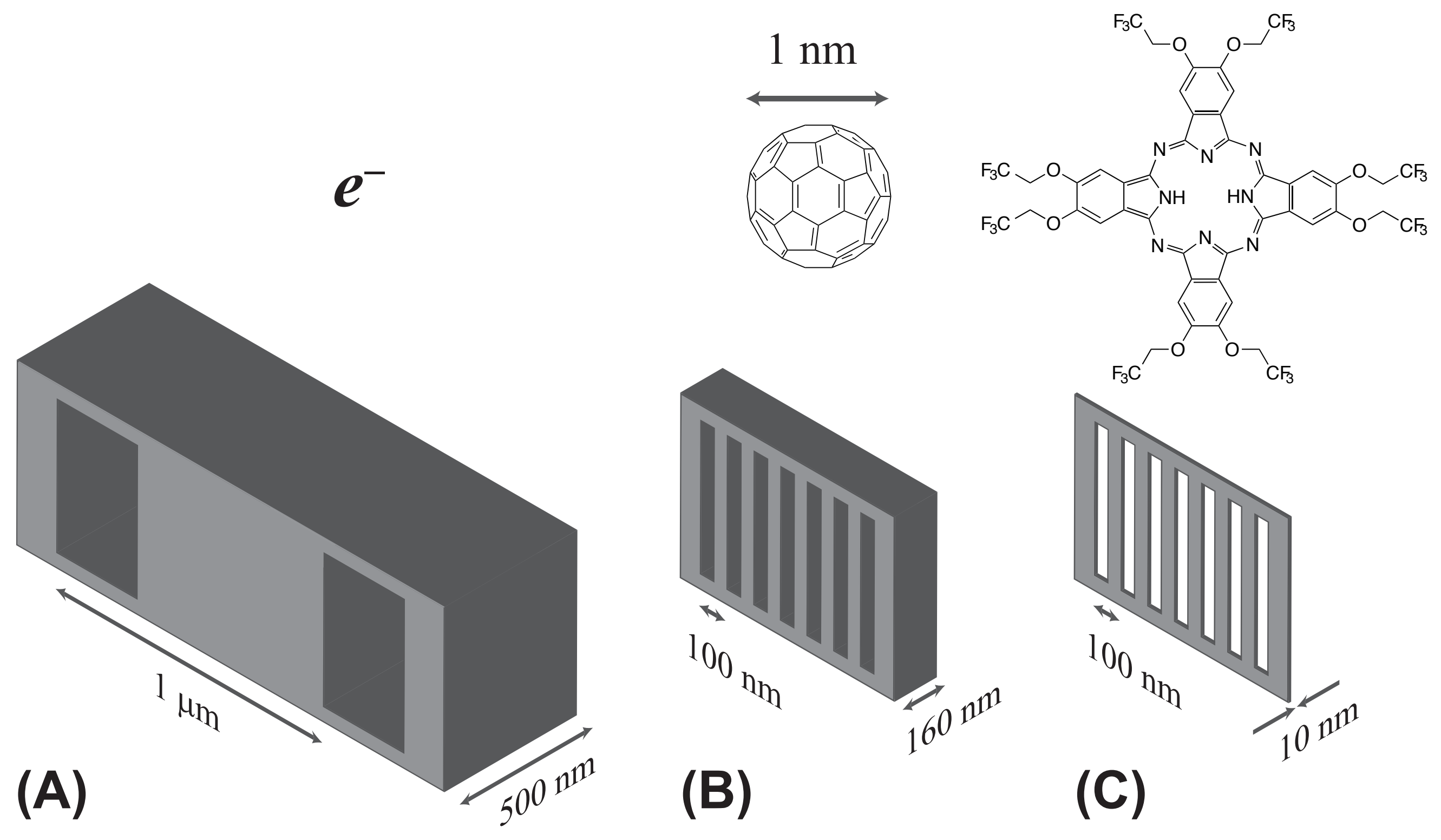

